\title{
Pineal Parenchymal Tumour of Intermediate Differentiation: a Case Report
}

\author{
Khaled A1, Joarder MA2, Chandy M³, Nasir TA4
}

\begin{abstract}
Pineal region tumours are rare, even though it gives rise to diverse array of tumour. Among these, pineal parenchymal tumour of intermediate differentiation is a newly recognized entity. We have described a 56 year old male of pineal parenchymal tumour of intermediate differentiation. As this tumour is very rare with uncertain behavior and optimal management, this case report will add value to the existing database.
\end{abstract}

\section{Introduction}

Pineal region tumours are rare, comprising $0.4 \%$ to $1 \%$ of adult brain tumours. Despite its small size, the pineal gland is the origin of a surprisingly diverse array of tumour types. The three major categories are germ cell tumours, parenchymal cell tumours and the supporting tissue neoplasm, (glial tumour). Germinomas are the most common histological type, comprising $50 \%-60 \%$ of all pineal tumours. ${ }^{1}$

Pineal parenchymal tumours represent about 30\% of pineal region neoplasm. ${ }^{2}$ The histogenesis of pineal parenchymal tumour is linked to the pineocytes, a cell with photosensory and neuroendocrine functions. Neoplasms derived from pineocytes or their embryonal precursors span a wide spectrum ranging from tumours composed of mature elements to ones composed of primitive, immature cells. Between these extremes are tumours demonstrating an intermediate degree of differentiation (PPTID). ${ }^{3}$

PPTID was recognized in the 2007 WHO classification as a new pineal parenchymal neoplasm, intermediate in malignancy (WHO grade-II and III) between pineocytoma (grade-I) and pineoblastoma (grade-IV). It occurs at all ages, including childhood to adult life with a peak incidence in early adults. ${ }^{3}$

There are very few reports about PPTID. So little is known about their clinical behavior and optimal management.4,5 Considering the above facts, we are presenting a case report of PPTID in

Apollo Hospitals Dhaka.

\section{Case Report}

A 56 year old normotensive, nondiabetic male attended the neurosurgery outpatient department with the complaint of vertigo for 3 months, vomiting for 4-5 days, blurring of vision and disorientation for 2 days.

On clinical examination, no significant findings were detected. Multiplanar and multisequence MRI (with contrast) and spectroscopy of the brain revealed a pineal mass with suggestion of intratumoural haemorrhage and intraventricular extension to third ventricle with mass effects causing obstructive hydrocephalous. After surgery, tissue from pineal mass sent for histopathological examination. Grossly, the tumour was composed of multiple small soft greyish brown pieces of tissue. Microscopic examination reveal proliferation of diffuse sheets and lobules of monomorphic, uniform, round cells with mild to moderate focal atypia (Fig 1), microvascular proliferation, focal calcification and presence of occasional tumour giant cells.

Immunohistochemistry for Neuron specific enolase (NSE) and glial fibrillary acidic protein (GFAP) shows positivity in tumour cells and supporting stroma. Based on location, histomorphology and immunohistochemical reactivity, pineal parenchymal tumour of intermediate differentiation (PPTD) diagnosis was made. 


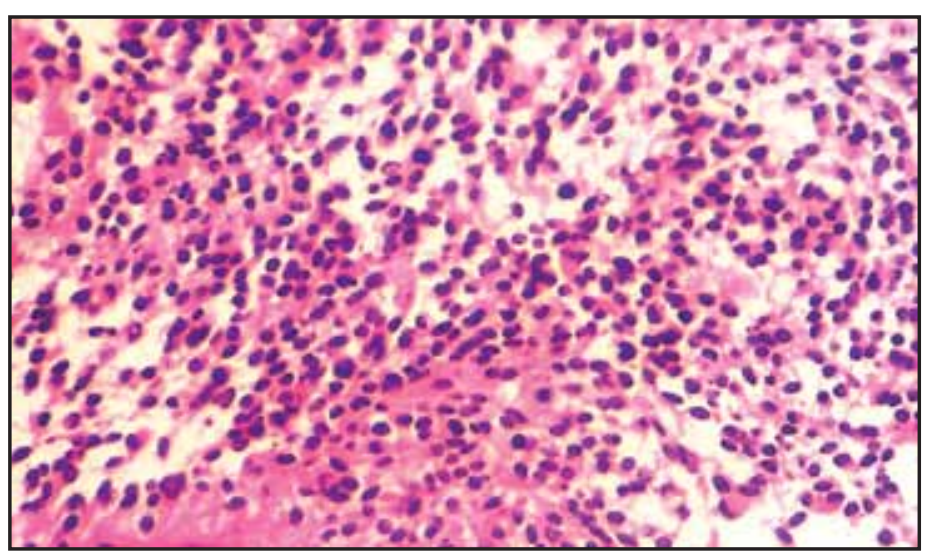

Fig 1: Proliferation of uniform round cells with focal mild to moderate atypia in PPTID

\section{Discussion}

PPTID comprises approximately $10 \%$ of all pineal parenchymal tumours. An accurate frequency of occurrence is unclear because of the diagnostic difficulties, both clinically and pathologically. ${ }^{6,7}$

The grading system of these tumours is also difficult and subjective. It has been suggested that a grading system based on mitotic activity and neurofilament protein immunoreactivity can differentiate low from high grade PPTID. ${ }^{7}$

According to this new prognostic grading PPTID can be graded into four grades, Grade-I for pineocytoma, Grade-IV for pineoblastoma and Grade-II being defined as having less than six mitotic figures per $10 \mathrm{HPF}$, Positive immunolabelling of neurofilament, and Grade-III being defined as having six or more mitotic figure per $10 \mathrm{HPF}$ and without immunostaining for neurofilament. ${ }^{7}$

We present this case of PPTID to enrich current database of this tumour. Diagnosing PPTID can be difficult and histopatholigic findings are not pathognomic. Besides an accurate grading is essential for these rare tumours, because optimal therapeutic management and prognosis depend on histopathological grading. ${ }^{2}$

\section{References}

1. Gasparetto EL, Warszawiak D, Adam GP, Bleggi-Torres LF, Neto AC. Glioblastoma multiforme of the pineal region: case report. Arq Neuropsiquiatr. 2003;61(2):468-472.

2. Kiyici H, Sanal B. Pineal parenchymal tumour of intermediate differentiation: a brief report. Eur J Basic Med Sci. 2012;2(1):30-33.

3. Mena H, Nakazato Y, Jouvet A. Pineal parenchymal tumour of intermediate differentiation. In Kleihues P, Cavenee WK, editors. Pathology and genetics of tumours of the Nervous system. Lyon: IARC Press; 2000. p. 115-121.

4. Pustaszeri M, Pica A, Janzer R. Pineal parenchymal tumours of intermediate differentiation in adults: case report and literature review. Neuropathology. 2006; 26:153-7.

5. Schild SE, Scheithauer BW, Schomberg PJ. Pineal parenchymal tumours. Clinical pathologic and therapeutic aspects. Cancer. 1993;72:870-880.

6. Fauchon F, Jouvet A, Paquis P. Parenchymal pineal tumours: a clinicopathological study of 76 cases. Int J Radiat Oncol Biol Phys. 2000;46:959-968.

7. Jouvet A, Saint- PierrG, Fauchon F. Pineal parenchymal tumours: a correlation of histological features with prognosis in 66 cases. Brain Pathol. 2000;10:49-60. 